Research Article

\title{
Prediction of dynamic stability behavior of thin square plates subjected to constant compressive and periodic including constant compressive loads on perpendicular edges
}

\author{
D.V. Ramana Reddy ${ }^{\dot{A}^{*}}$, B. Subba Ratnam ${ }^{\dot{\mathrm{B}}}$, G. Venkateswara Rao ${ }^{\dot{\mathrm{A}}}$ \\ ${ }^{\AA}$ Department of Mechanical Engineering, Vardhaman College of Engineering, Shamshabad, Hyderabad-501218, India, \\ ${ }^{\mathrm{b}}$ Department of Mechanical Engineering, Krishnachaitanya Institute of Technology and Sciences - 523 320, India,
}

Accepted 10 January 2014, Available online 01 February 2014, Special Issue-2, (February 2014)

\begin{abstract}
The dynamic stability behavior of the square plate subjected to a uniform edge in-plane periodic load, consisting of a constant compressive and periodic load in one direction and a constant compressive load in the perpendicular direction is investigated for the simply supported boundary conditions with varying uniform constant compressive loads, in this paper. The energy method, by using a single term exact trigonometric admissible function to represent the lateral deflection, is employed to obtain the dynamic instability regions. Numerical results are presented in the non-dimensional form with the varying static load parameters and the static compressive load ratios in both the digital and analogue forms. The present results show excellent agreement when compared with that available in the literature. The effect of the static load parameters and the static compressive load ratios on the dynamic instability regions is brought out in the present work.
\end{abstract}

Keywords: Dynamic stability; simple formula; thin square plate; periodic load; constant loads

\section{Introduction}

The present day lightweight structures, subjected to dynamic loads, make it essential to determine the dynamically unstable regions for different structural members. When a plate is subjected to periodic loads, it is a well known fact that for certain values of exciting frequencies the ordinary in-plane forced response will become dynamically unstable, leading to a violent vibration in the transverse direction. This is called as the dynamic instability phenomenon.

Prediction of the dynamic stability behavior of structural members subjected to periodic in-plane loads is an important input for the structural design engineers. The structural members, like plates are commonly used in many fields of engineering. Earlier studies on the dynamic stability of columns are briefly discussed (Timoshenko and Gere, 1961). The theory and application of the dynamic stability behavior of structures have been exhaustively given in the classic work of Bolotin (Bolotin, 1964). The first finite element (FE) studies on this topic for columns with various boundary conditions (Brown et al. 1968). It is observed in this interesting study that when the first mode shapes of the free vibration and buckling are the same or nearly the same, which is generally satisfied for the many column boundary conditions, the dynamic stability regions, more or less remain the same, by proper

*Corresponding author: D.V. Ramana Reddy non-dimensionalization of the basic physical quantities in the problem involved. However, the derivation of the proper nondimensional parameters is not given in the study and is taken intuitively (Brown et al. 1968). The thin plate finite element model to study the dynamic instability of rectangular plates studied in this work (Hutt and Salama, 1971). Some recent studies in this topic for plates subjected to periodic loading can be seen in (Dey and Singha, 2006), (Ramachandran and Sarat Kumar, 2012). In a recent study (Rao et al, 2008), it is shown that these nondimensional parameters used in (Brown et al. 1968), can be derived rigorously and demonstrated the existence of the unique dynamic stability curves for many commonly used structural members, provided the requirement on the mode shapes (Brown et al. 1968) is satisfied. It may be emphasized here that, these mode shapes, though similar as mentioned earlier (Brown et al. 1968), differ by small extent depending on the boundary conditions and the error involved in the instability boundaries is tolerable small, for the engineering purposes, as has already been demonstrated by (Rao et al, 2011), based on the consideration of the Euclidean norm.

In the present study, the dynamic stability behavior of the square plate subjected to an edge uniform in-plane periodic load, consisting of a constant compressive and periodic loads in one direction and a constant compressive load in the perpendicular direction, is investigated for the plate with the simply supported boundary conditions with varying uniform constant compressive load ratios. In this 
work an energy method is used to develop a simple closed form solution to predict the dynamic instability behavior of the simply supported square plate, using exact trigonometric admissible function to represent the transverse deflection, as given by (Leissa 1965).

Elegant, simple and exact closed form analytical solution to predict the dynamic stability regions of the square plate subjected to the aforementioned loads are provided in this paper. It is to be noted here, that the prediction of dynamic stability behavior of the plate with these applied loads is a new contribution, to obtain the behavior of dynamic stability regions of square plate. In the following section, a simple formula to predict the dynamic instability behavior of the simply supported square plate subjected to the loading condition, mentioned earlier is presented.

\section{Dynamic Stability Equation}

When the plate is, subjected to a uniform edge in-plane periodic load in the $\mathrm{x}$ - direction and constant compressive load in the $y$ - direction, as shown in Fig.1, the periodic $\operatorname{load} \mathrm{N}_{\mathrm{x}}(\mathrm{t})$ and $\mathrm{N}_{\mathrm{y}}$, which are defined as,

$$
\begin{aligned}
& N_{x}(t)=N_{s}+N_{t} \cos \theta t=\left(\alpha \pm \beta \cos \theta t_{t}\right) N_{c r} \\
& \text { and } \\
& N_{y}=\gamma N_{x}
\end{aligned}
$$

where $\alpha$ is the static load factor $\left(=\frac{N_{s}}{N_{c r}}\right), \beta$ is the dynamic load factor $\left(=\frac{N_{t}}{N_{c r}}\right), N_{s}$ is the constant compressive load,

$N_{t}$ is the time dependent load, $N_{c r}$ is the buckling (critical) load, $\theta$ is the applied radian frequency, $t_{t}$ indicates time and $\gamma=\frac{N_{x}}{N_{y}}$.

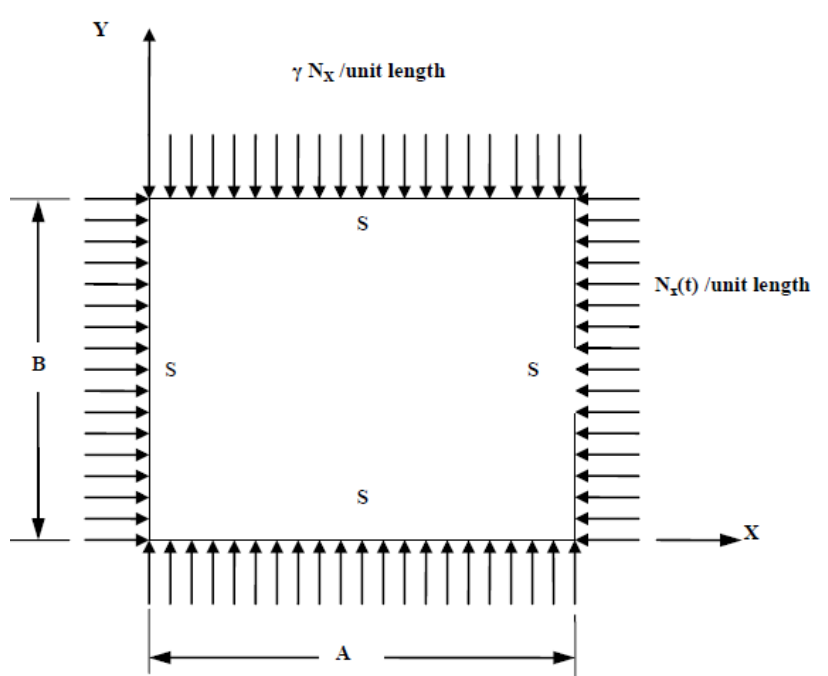

Fig.1 A simply supported plate under the applied compressive load system

For a rectangular plate of length $A$ and breadth $B$ with constant thickness $t$, the strain energy $U$, the work done by the external compressive edge loads $W$ and the kinetic energy $T$ are given by

$$
\begin{gathered}
U=\frac{D}{2} \int_{0}^{B} \int_{0}^{A}\left[\kappa_{x}^{2}+\kappa_{y}^{2}+2 v \kappa_{x} \kappa_{y}+\frac{1-v}{2} \kappa_{x y}^{2}\right] d x d y(3) \\
W=\frac{N_{x}(t)}{2} \int_{0}^{B} \int_{0}^{A}\left(\frac{\partial w}{\partial x}\right)^{2} d x d y+\frac{\gamma N_{x}}{2} \int_{0}^{B A} \int_{0}^{A}\left(\frac{\partial w}{\partial y}\right)^{2} d x d y(4)
\end{gathered}
$$

and

$$
T=\frac{\rho t \omega^{2}}{2} \int_{0}^{B} \int_{0}^{A} w^{2} d x d y
$$

with

$\kappa_{x}=-\frac{\partial^{2} w}{\partial x^{2}}, \kappa_{y}=-\frac{\partial^{2} w}{\partial y^{2}}, \kappa_{x y}=-2 \frac{\partial^{2} w}{\partial x \partial y}$

Where $w$ is the lateral displacement, $\rho$ is the mass density, $\omega$ is the radian frequency of the plate, $N_{x}(t)$ and $N_{y}$ are the edge uniform periodic and constant compressive loads in the Cartesian co-ordinate system, and $D$ is the plate flexural rigidity given by $D=\frac{E t^{3}}{12\left(1-v^{2}\right)}, \mathrm{E}$ is the young's modulus and $v$ is the Poisson ratio.

The admissible transverse displacement $w$ in terms of $m$ and $n$, which are the number of half-waves in the $x$ and $y$ directions respectively, is assumed to be of the form,

$$
w=a \sin \left(\frac{m \pi x}{A}\right) \sin \left(\frac{n \pi y}{B}\right)
$$

Where $a$ is the undetermined coefficient. The displacement distribution assumed for $w$ is exact for the simply supported boundary conditions, for both the vibration and buckling problems.

The total potential energy $\Pi$ of the plate is given by

$$
\Pi=U-W-T
$$

$$
\begin{aligned}
& \Pi=\left(\begin{array}{l}
\frac{D}{2} \int_{0}^{B A} \int_{0}^{A}\left[\kappa_{x}^{2}+\kappa_{y}^{2}+2 v \kappa_{x} \kappa_{y}+\frac{1-v}{2} \kappa_{x y}^{2}\right] d x d y- \\
\frac{\gamma N_{x}}{2} \int_{0}^{B A} \int_{0}^{A}\left(\frac{\partial w}{\partial y}\right)^{2} d x d y
\end{array}\right)- \\
& \frac{N_{x}(t)}{2} \int_{0}^{B} \int_{0}^{A}\left(\frac{\partial w}{\partial x}\right)^{2} d x d y
\end{aligned}
$$

Where $U, T$ and $W$ are the strain energy, kinetic energy and potential due to the work respectively.

In the Rayleigh-Ritz method, the total potential energy is minimized with respect to the undetermined coefficient $a$, as

$$
\frac{\partial \Pi}{\partial a}=\left(\frac{\partial}{\partial a}\right)(U-W-T)=0
$$

Which yields, after integration and simplification of Eq.(10) by neglecting the term for $W$, an expression for the frequency parameter, $\lambda_{f}\left(=\frac{\rho t \omega^{2} A^{4}}{D}\right)$ is obtained as

$$
\lambda_{f}=\pi^{4}\left(m^{2}+n^{2} \frac{A^{2}}{B^{2}}\right)^{2}
$$

Similarly, by neglecting the kinetic energy term $T$ in Eq.(10), the expression for the buckling load parameter, $\lambda_{b}$ 
( $=\frac{N_{x c r} A^{2}}{\pi^{2} D}$, where $N_{x c r}$ is the critical load, defined with the load in the $\mathrm{x}$ - direction, is obtained as

$$
\lambda_{b}=\frac{\left(m^{2}+n^{2} \frac{A^{2}}{B^{2}}\right)^{2}}{\left(m^{2}+m^{2} \frac{A^{2}}{B^{2}}\right)}
$$

Substituting, the energies $U$ and $T$ and potential $W$ in Eq. (10), after simplification, using the Eqs.(11) and Eq.(12) for, the dynamic stability equation in the non-dimensional form, as

$$
1-\frac{\left\{\left(\alpha \pm \frac{\beta}{2}\right) m^{2}+m^{2} \frac{A^{2}}{B^{2}}\right\}}{\left(m^{2}+m^{2} \frac{A^{2}}{B^{2}}\right)}-\frac{\theta^{2}}{4 \omega^{2}}=0
$$

For a square plate $(\mathrm{A} / \mathrm{B}=1)$, for the first mode of buckling and vibration $(m=n=1)$ and $\gamma=0$ for uni-axial load, Eq. (13) becomes

$$
1-\left(\alpha \pm \frac{\beta}{2}\right)-\frac{\theta^{2}}{4 \omega^{2}}=0
$$

Defining, $\Omega=\frac{\theta}{\omega} \quad$ and $\quad$ after simplification, Eq.

becomes

$$
\Omega=\frac{\theta}{\omega}=2 \sqrt{1-\left(\alpha \pm \frac{\beta}{2}\right)}
$$

Equation (15) can be treated as the dynamic stability solution containing the physically identifiable nondimensional parameters.

Substituting, the energies $U$ and $T$ and potential $W$ in Eq. (10), after simplification, using the Eqs.(11) and Eq.(12) for biaxial load, and after simplification, the dynamic stability equation in the non-dimensional form, as

$\Omega=\frac{\theta}{\omega}=2 \sqrt{1-\frac{1}{2}\left\{\left(\alpha \pm \frac{\beta}{2}\right)+\gamma\right\}}$

Equation (16) can be treated as the dynamic stability solution for the afore mentioned loading condition, containing the physically identifiable non-dimensional parameters.

\section{Results and Discussion}

By using Eq. (15), the dynamic stability of square plate with uni axial load and using Eq.(16) the effect of the inplane loads considered, in the present study, as shown in Fig.1, is brought out. The dynamic instability boundaries $\Omega_{1}$ and $\Omega_{2}$, between which it is dynamically unstable are given with varying $\beta$ and for $\alpha=0.0,0.25$ and 0.5 given in Table 1. In Table.1, the variation of $\Omega_{1}$ and $\Omega_{2}$ for the value of $\beta$ (dynamic load factor) varying from 0 to 1.0 for the simply supported square plate with uniform uniaxial in-plane periodic load i.e. $(\gamma=0.0)$ for $\alpha=0.0,0.25$ and 0.5 are presented. The dynamic stability regions of the square plate are given in (Ramachandra and Sarat Kumar, 2012) and the present results for $\gamma=0.0$ (Uniform uniaxial periodic load) are deduced from the dynamic stability formulae Eq.(15), derived here, for $\alpha=0.0$ and 0.6 as is given in Tables 2 and 3 respectively. The excellent agreement between the two results strongly indicates the usefulness of the simple dynamic stability formula, developed in the present work.

Table 1: Variation of $\Omega_{1}$ and $\Omega_{2}$ for square plate subjected to uniaxial compression without static compressive load $(\gamma=0.0)$

\begin{tabular}{|l|l|l|l|l|l|l|}
\hline \multirow{2}{*}{$\boldsymbol{\beta}$} & \multicolumn{2}{|l|}{$\boldsymbol{\alpha}=\mathbf{0 . 0}$} & \multicolumn{2}{l}{$\boldsymbol{\alpha}=\mathbf{0 . 2 5}$} & \multicolumn{2}{l|}{$\boldsymbol{\alpha}=\mathbf{0 . 5}$} \\
\cline { 2 - 7 } & $\mathbf{\Omega}_{\mathbf{1}}$ & $\mathbf{\Omega}_{\mathbf{2}}$ & $\mathbf{\Omega}_{\mathbf{1}}$ & $\mathbf{\Omega}_{\mathbf{2}}$ & $\mathbf{\Omega}_{\mathbf{1}}$ & $\mathbf{\Omega}_{\mathbf{2}}$ \\
\hline 0.0 & 2.0000 & 2.0000 & 1.7320 & 1.7320 & 1.4142 & 1.4142 \\
\hline 0.1 & 1.9493 & 2.0493 & 1.6733 & 1.7888 & 1.3416 & 1.4832 \\
\hline 0.2 & 1.8973 & 2.0976 & 1.6124 & 1.8439 & 1.2649 & 1.5491 \\
\hline 0.3 & 1.8473 & 2.1447 & 1.5491 & 1.8973 & 1.1832 & 1.6124 \\
\hline 0.4 & 1.7888 & 2.1908 & 1.4832 & 1.9493 & 1.0954 & 1.6733 \\
\hline 0.5 & 1.7320 & 2.2360 & 1.4142 & 2.0000 & 1.0000 & 1.7320 \\
\hline 0.6 & 1.6733 & 2.2803 & 1.3416 & 2.0493 & 0.8944 & 1.7888 \\
\hline 0.7 & 1.6124 & 2.3237 & 1.2649 & 2.0976 & 0.7745 & 1.8439 \\
\hline 0.8 & 1.5491 & 2.3664 & 1.1832 & 2.1447 & 0.6324 & 1.8973 \\
\hline 0.9 & 1.4832 & 2.4083 & 1.0954 & 2.1908 & 0.4472 & 1.9493 \\
\hline 1.0 & 1.4142 & 2.4493 & 1.0000 & 2.2360 & 0.0000 & 2.0000 \\
\hline
\end{tabular}

Table 2: Variation of $\Omega_{1}$ and $\Omega_{2}$ for square plate Subjected to uniaxial periodic load for $\alpha=0.0$

\begin{tabular}{|l|l|l|l|l|}
\hline \multirow{2}{*}{$\beta$} & \multicolumn{2}{|l}{ Present study } & $\begin{array}{l}\text { Ramachandra } \\
\text { Kumar }^{*}\end{array}$ \\
\cline { 2 - 5 } & $\boldsymbol{\Omega}_{\mathbf{1}}$ & $\mathbf{\Omega}_{\mathbf{2}}$ & $\mathbf{\Omega}_{\mathbf{1}}$ & $\boldsymbol{\Omega}_{\mathbf{2}}$ \\
\hline 0.0 & 39.4801 & 39.4801 & 39.09 & 39.09 \\
\hline 0.1 & 38.4790 & 40.4531 & 38.63 & 40.00 \\
\hline 0.2 & 37.4527 & 41.4066 & 37.27 & 40.91 \\
\hline 0.3 & 36.4657 & 42.3363 & 36.59 & 42.04 \\
\hline 0.4 & 35.3109 & 43.2463 & 35.22 & 42.73 \\
\hline 0.5 & 34.1890 & 44.1386 & 34.54 & 43.64 \\
\hline 0.6 & 33.0309 & 45.0131 & 32.72 & 44.54 \\
\hline 0.7 & 31.8287 & 45.8698 & 31.81 & 45.45 \\
\hline 0.8 & 30.5792 & 46.7127 & 30.68 & 46.36 \\
\hline 0.9 & 29.2783 & 47.5398 & 29.45 & 46.81 \\
\hline 1.0 & 27.9163 & 48.3491 & 27.27 & 47.72 \\
\hline \multicolumn{7}{|l|}{ *alues are read from the graph } \\
\hline
\end{tabular}

Table 3: Variation of $\Omega_{1}$ and $\Omega_{2}$ for square plate subjected to uniaxial periodic load for $\alpha=0.6$

\begin{tabular}{|l|l|l|l|l|}
\hline \multirow{2}{*}{$\beta$} & \multicolumn{2}{|l|}{ Present Formula } & \multicolumn{2}{l|}{$\begin{array}{l}\text { Ramachandra } \\
\text { Sarat Kumar }\end{array}$} \\
\cline { 2 - 5 } & $\boldsymbol{\Omega}_{\mathbf{1}}$ & $\mathbf{\Omega}_{\mathbf{2}}$ & $\mathbf{\Omega}_{\mathbf{1}}$ & $\mathbf{\Omega}_{\mathbf{2}}$ \\
\hline 0.0 & 24.9693 & 24.9693 & 25.03 & 25.03 \\
\hline 0.05 & 24.1755 & 25.7370 & 24.18 & 25.64 \\
\hline 0.1 & 23.3563 & 26.4831 & 23.45 & 26.36 \\
\hline 0.15 & 22.5055 & 27.2096 & 22.48 & 27.09 \\
\hline 0.2 & 21.6231 & 27.9163 & 21.51 & 27.93 \\
\hline 0.25 & 20.7033 & 28.6052 & 20.79 & 28.54 \\
\hline 0.3 & 19.7393 & 29.2783 & 19.81 & 29.27 \\
\hline
\end{tabular}

Table 4: Variation of $\Omega_{1}$ and $\Omega_{2}$ for square plate subjected to applied compressive load system with static compressive load ratio $(\gamma=0.25)$

\begin{tabular}{|l|l|l|l|l|l|l|}
\hline \multirow{3}{*}{$\beta$} & \multicolumn{3}{|l|}{$\alpha=0.0$} & \multicolumn{2}{l|}{$\alpha=0.25$} & \multicolumn{2}{l|}{$\alpha=0.5$} \\
\cline { 2 - 7 } & $\Omega_{1}$ & $\Omega_{2}$ & $\Omega_{1}$ & $\Omega_{2}$ & $\Omega_{1}$ & $\Omega_{2}$ \\
\hline
\end{tabular}

622 | International Conference on Advances in Mechanical Sciences 2014 


\begin{tabular}{|l|l|l|l|l|l|l|}
\hline 0.0 & 1.8708 & 1.8708 & 1.7320 & 1.7320 & 1.5811 & 1.5811 \\
\hline 0.1 & 1.8439 & 1.8973 & 1.7029 & 1.7608 & 1.5491 & 1.6124 \\
\hline 0.2 & 1.8165 & 1.9235 & 1.6733 & 1.7888 & 1.5165 & 1.6431 \\
\hline 0.3 & 1.7829 & 1.94938 & 1.6431 & 1.8165 & 1.4832 & 1.6733 \\
\hline 0.4 & 1.7606 & 1.9748 & 1.6124 & 1.8439 & 1.4491 & 1.7029 \\
\hline 0.5 & 1.732 & 2.0000 & 1.5811 & 1.8708 & 1.4142 & 1.732 \\
\hline 0.6 & 1.7029 & 2.0248 & 1.5491 & 1.8973 & 1.3784 & 1.7606 \\
\hline 0.7 & 1.6733 & 2.0493 & 1.5165 & 1.9235 & 1.3416 & 1.7888 \\
\hline 0.8 & 1.6431 & 2.0736 & 1.4832 & 1.9493 & 1.3038 & 1.8165 \\
\hline 0.9 & 1.6124 & 2.0976 & 1.4491 & 1.9748 & 1.2649 & 1.8439 \\
\hline 1.0 & 1.5811 & 2.1213 & 1.4142 & 2.0000 & 1.2247 & 1.8708 \\
\hline
\end{tabular}

Table 5: Variation of $\Omega_{1}$ and $\Omega_{2}$ for square plate subjected to applied compressive load system with static compressive load ratio $(\gamma=0.5)$

\begin{tabular}{|l|l|l|l|l|l|l|}
\hline \multirow{3}{*}{$\boldsymbol{\beta}$} & \multicolumn{2}{|l|}{$\boldsymbol{\alpha}=\mathbf{0 . 0}$} & \multicolumn{2}{l|}{$\boldsymbol{\alpha}=\mathbf{0 . 2 5}$} & \multicolumn{2}{l|}{$\boldsymbol{\alpha}=\mathbf{0 . 5}$} \\
\cline { 2 - 7 } & $\boldsymbol{\Omega}_{\mathbf{1}}$ & $\boldsymbol{\Omega}_{\mathbf{2}}$ & $\boldsymbol{\Omega}_{\mathbf{1}}$ & $\boldsymbol{\Omega}_{\mathbf{2}}$ & $\boldsymbol{\Omega}_{\mathbf{1}}$ & $\boldsymbol{\Omega}_{\mathbf{2}}$ \\
\hline 0.0 & 1.4142 & 1.4142 & 1.2247 & 1.2247 & 1.0000 & 1.0000 \\
\hline 0.1 & 1.3784 & 1.4491 & 1.1832 & 1.2649 & 0.9486 & 1.0487 \\
\hline 0.2 & 1.3416 & 1.4832 & 1.1401 & 1.3038 & 0.8944 & 1.0954 \\
\hline 0.3 & 1.3038 & 1.5165 & 1.0954 & 1.3416 & 0.8366 & 1.1401 \\
\hline 0.4 & 1.2649 & 1.5491 & 1.0488 & 1.3784 & 0.7745 & 1.1832 \\
\hline 0.5 & 1.2247 & 1.5811 & 1.0000 & 1.4142 & 0.7071 & 1.2247 \\
\hline 0.6 & 1.1832 & 1.6124 & 0.9486 & 1.4491 & 0.6324 & 1.2648 \\
\hline 0.7 & 1.1401 & 1.6431 & 0.8944 & 1.4832 & 0.5477 & 1.3038 \\
\hline 0.8 & 1.0954 & 1.6733 & 0.8366 & 1.5165 & 0.4472 & 1.3416 \\
\hline 0.9 & 1.0488 & 1.7029 & 0.7745 & 1.5491 & 0.3162 & 1.3784 \\
\hline 1.0 & 1.0000 & 1.732 & 0.7071 & 1.5811 & 0.0000 & 1.4142 \\
\hline
\end{tabular}

Table 6: Variation of $\Omega_{1}$ and $\Omega_{2}$ for square plate subjected to applied compressive load system with static compressive load ratio $(\gamma=1.0)$

\begin{tabular}{|l|l|l|l|l|l|l|}
\hline \multirow{2}{*}{$\boldsymbol{\beta}$} & \multicolumn{3}{|l|}{$\boldsymbol{\alpha}=\mathbf{0 . 0}$} & \multicolumn{2}{l|}{$\boldsymbol{\alpha}=\mathbf{0 . 2 5}$} & \multicolumn{2}{l|}{$\boldsymbol{\alpha}=\mathbf{0 . 5}$} \\
\cline { 2 - 7 } & $\mathbf{\Omega}_{\mathbf{1}}$ & $\mathbf{\Omega}_{\mathbf{2}}$ & $\mathbf{\Omega}_{\mathbf{1}}$ & $\mathbf{\Omega}_{\mathbf{2}}$ & $\mathbf{\Omega}_{\mathbf{1}}$ & $\mathbf{\Omega}_{\mathbf{2}}$ \\
\hline 0.0 & 1.8708 & 1.8708 & 1.7320 & 1.7320 & 1.5811 & 1.5811 \\
\hline 0.1 & 1.8439 & 1.8973 & 1.7029 & 1.7608 & 1.5491 & 1.6124 \\
\hline 0.2 & 1.8165 & 1.9235 & 1.6733 & 1.7888 & 1.5165 & 1.6431 \\
\hline 0.3 & 1.7829 & 1.94938 & 1.6431 & 1.8165 & 1.4832 & 1.6733 \\
\hline 0.4 & 1.7606 & 1.9748 & 1.6124 & 1.8439 & 1.4491 & 1.7029 \\
\hline 0.5 & 1.732 & 2.0000 & 1.5811 & 1.8708 & 1.4142 & 1.732 \\
\hline 0.6 & 1.7029 & 2.0248 & 1.5491 & 1.8973 & 1.3784 & 1.7606 \\
\hline 0.7 & 1.6733 & 2.0493 & 1.5165 & 1.9235 & 1.3416 & 1.7888 \\
\hline 0.8 & 1.6431 & 2.0736 & 1.4832 & 1.9493 & 1.3038 & 1.8165 \\
\hline 0.9 & 1.6124 & 2.0976 & 1.4491 & 1.9748 & 1.2649 & 1.8439 \\
\hline 1.0 & 1.5811 & 2.1213 & 1.4142 & 2.0000 & 1.2247 & 1.8708 \\
\hline
\end{tabular}

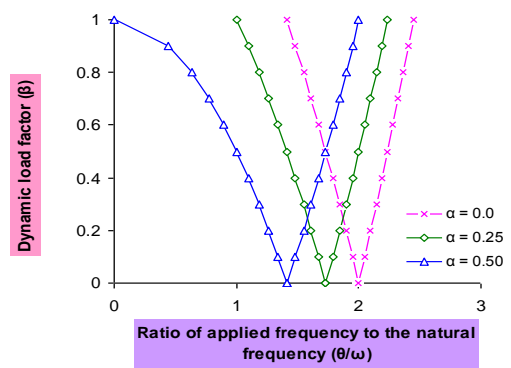

Fig. 2 Dynamic stability regions for square plate subjected to uniaxial compression without static compressive load $(\gamma=0.0)$

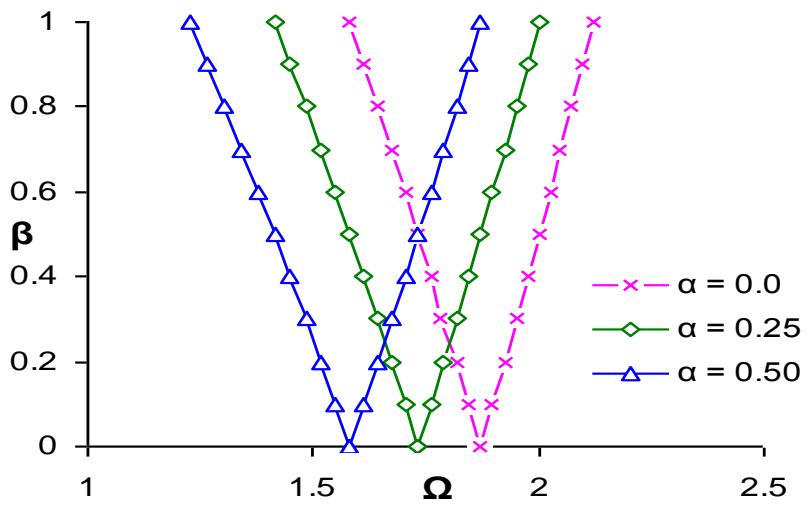

Fig. 3 Dynamic stability curves for square plate subjected to the applied compressive load system with static compressive load ratio $(\gamma=0.25)$

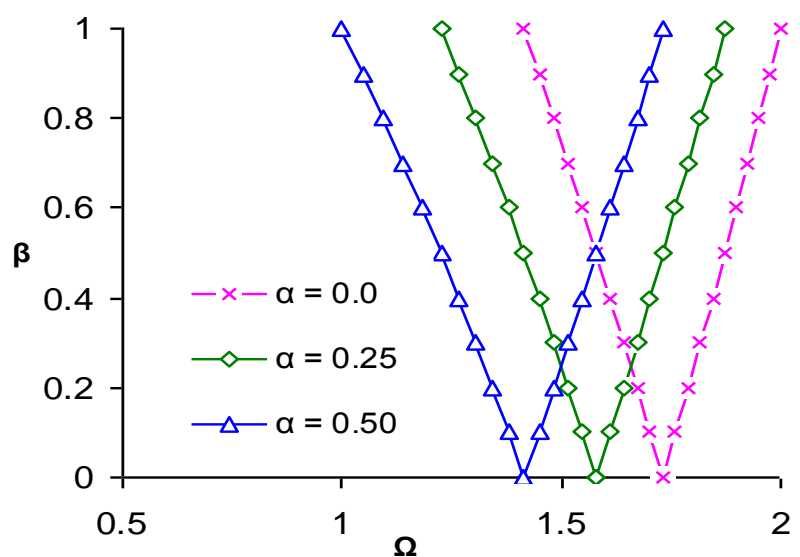

Fig. 4 Dynamic stability curves for square plate subjected to the applied compressive load system with static compressive load rati $(\gamma=0.5)$

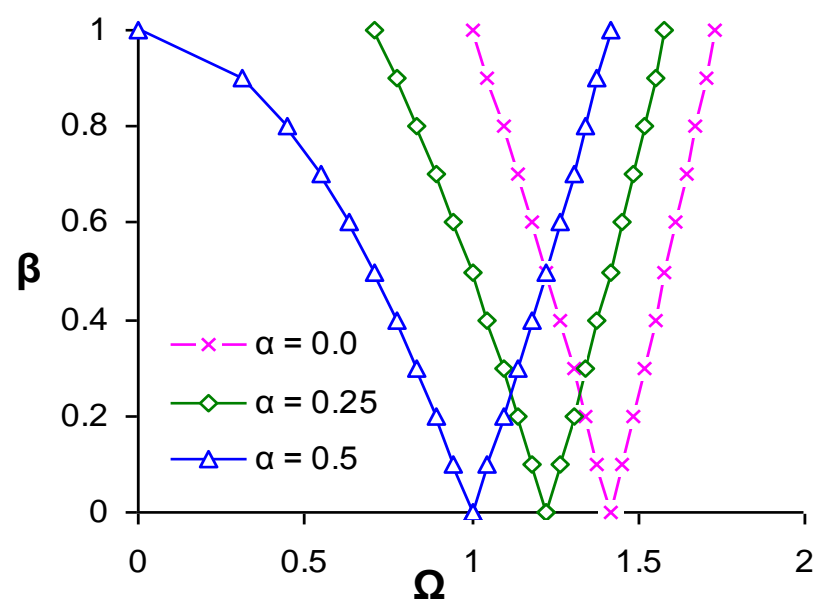

Fig. 5 Dynamic stability curves for square plate subjected to the applied compressive load system with static compressive load ratio $(\gamma=1.0)$

In Tables 4 to 6 , the dynamic stability regions for the uniform biaxial load for $\gamma=0.25,0.5$ and 1.0 for $\alpha=0.0$, 0.25 and 0.5 are presented. Figures 3 to 5 shows the stability boundaries given in tables 4 to 6 respectively. One can observe that, by increasing of the static compressive load parameter $\alpha$, the width of the dynamic instability regions increase and by increasing the static 
compressive load ratio $(\gamma)$ the regions of the dynamic instability increases and shifts towards the vertical axis.

\section{Conclusions}

Accurate closed form solutions are obtained to predict the dynamic stability regions of the simply supported square plate subjected to uniform in-plane compressive periodic load with a constant compressive load component in one direction and a static compressive load in the perpendicular direction. Simple one term standard trigonometric admissible function that satisfies all the boundary conditions is used to obtain the solution employing the energy method. It is noted here, by increasing the static load factor $\alpha$, the regions of instability increase and by increasing the static compressive load ratio $(\gamma)$ the dynamic instability regions increases and shift towards the vertical axis using physically recognizable dynamic load factor $(\beta)$.

\section{Acknowledgements}

The authors are grateful to the Managements of their respective Institutes for their encouragement during the course of this work.

\section{References}

S. P. Timoshenko and J. M. Gere,(196), Theory of Elastic Stability, Mc Graw- Hill book Company Inc., New York.

V.V. Bolotin, (1964), Dynamic Stability of Elastic Systems. HoldenDay, San Francisco.

J.E. Brown, J. M. Hutt and A.E. Salama,(1968) Finite element solution to dynamic stability of bars, AIAA J, vol. 6, pp.14231425 .

J. M. Hutt and A.E. Salama, (1971) Dynamic stability of plates by finite elements. Proc. ASCE, EM.-97, pp.879-899

Dey, P., and Singha, M.K (2006) Dynamic Stability Analysis of Composite Skew Plates Subjected to

Periodic In-plane Load, Thin-Walled Structures, Vol.44, No.9, pp. 937-942

Ramachandra, L.S., and Sarat Kumar Panda, (2012) Dynamic instability of composite plates subjected to non-uniform in-plane loads", Journal of Sound and Vibrations, Vol.331, pp.53-65.

G. V. Rao, B.S. Ratnam and G.R. Janardhana, (2008) Master Dynamic Stability Formula for Structural members Subjected to Periodic Loads, AIAA Journal, 46, 537-540

G.V. Rao, B.S. Ratnam, Jagadish Babu Gunda and G.R. Janardhana, (2011) Master Formula for Evaluating Vibration Frequencies of Structural Members under Compressive Loads, The IES Journal Part A: Civil \& Structural Engineering, Vol.4, No.2, pp.79-88.

Leissa, A.W., (1965) Vibration of Plates, NASA SP-160, Washington D.C. 